\title{
A New Method of Estimating the Asset Rate of Return
}

\author{
Moawia Alghalith, Tracy Polius \\ Economics, University of the West Indies, St Augustine, USA \\ E-mail: malghalith@gmail.com, tracy.polius@sta.uwi.edu \\ Received April 19, 2011; revised April 21, 2011; accepted April 25, 2011
}

\begin{abstract}
\end{abstract}
We present a new consumption-based method of estimating the asset rate of return.

Keywords: Return, Investment, Portfolio, Asset, Stochastic, Consumption CAPM

In this note, we present a new model that links the stock/portfolio rate of return to consumption. Our approach is more general than the existing models such as the consumption-CAPM models, that are based on very restrictive assumptions [1]. In so doing, we utilize a more advanced and appropriate theoretical and empirical framework than the ones used by previous literature. It is worth noting that previous literature mainly used simple linear regressions without a rigorous theoretical basis.

We use a stochastic factor model, which includes a risky asset (portfolio, a risk-free asset and a stochastic external economic factor $[2,3]$. Thus, we have a twodimensional standard Brownian motion

$\left\{\left(W_{1 s}, W_{2 s}\right), \mathcal{F}_{s}\right\}_{t \leq s \leq T}$ on the probability space $\left(\Omega, \mathcal{F}_{s}, P\right)$, where $\left\{\mathcal{F}_{s}\right\}_{t \leq s \leq T}$ is the augmentation of filtration. The risk-free asset price process is $S_{0}=\mathrm{e}^{\int^{t} r\left(Z_{s}\right) \mathrm{ds}}$, where $r\left(Z_{s}\right) \in C_{b}^{2}(R)$, is the rate of return and $Z_{s}$ is the stochastic economic factor.

The dynamics of the risky asset price is given by

$$
\mathrm{d} S_{s}=S_{t}\left\{\mu\left(Z_{s}\right) \mathrm{d} t+\sigma\left(Z_{s}\right) \mathrm{d} W_{s}^{1}\right\},
$$

where $\mu\left(Z_{t}\right)$ and $\sigma\left(Z_{t}\right)$ are the rate of return and the volatility, respectively. The stochastic economic factor process is defined as

$$
\mathrm{d} Z_{s}=a\left(z_{s}\right) \mathrm{d} t+\rho \mathrm{d} W_{1 s}+\gamma \sqrt{1-\rho^{2}} \mathrm{~d} W_{2 s}, Z_{t}=z,
$$

where $|\rho|<1$ is the correlation factor between the two Brownian motions, $\gamma$ is a parameter, and $a\left(Z_{s}\right) \in C^{1}(R)$ has a bounded derivative.

The wealth process is given by

$$
\begin{aligned}
X_{T}^{\pi, c}= & x+\int_{t}^{T}\left\{r\left(Y_{s}\right) X_{s}^{\pi, c}+\left(\mu\left(Y_{s}\right)-r\left(Y_{s}\right) \pi_{s}\right)-c_{s}\right\} \mathrm{d} s \\
& +\int_{t}^{T} \pi_{s} \sigma\left(Y_{s}\right) \mathrm{d} W_{s}^{1},
\end{aligned}
$$

where $x$ is the initial wealth, $\left\{\pi_{t}, \mathcal{F}_{s}\right\}_{t \leq s \leq T}$ is the portfolio process and $\left\{c_{t}, \mathcal{F}_{s}\right\}_{t \leq s \leq T}$ is the consumption process, with $\int_{t}^{T} \pi_{s}^{2} \mathrm{~d} s<\infty, \int_{t}^{T} c_{s} \mathrm{~d} s<\infty$ and $c \geq 0$. The trading strategy $\left(\pi_{s}, c_{s}\right) \in A(x, y)$ is admissible.

The investor's objective is to maximize the expected utility of terminal wealth and consumption

$$
v(t, x, y)=\operatorname{Sup}_{\pi_{t}, c_{t}} E\left[U_{1}\left(X_{T}^{\pi, c}\right)+\int_{t}^{T} U_{2}\left(c_{s}\right) \mathrm{d} s \mid \mathcal{F}_{t}\right],
$$

where $v($.$) is the (smooth) value function, U($.$) ,$ bounded and strictly concave utility function.

The value function satisfies the Hamiltonian-JacobiBellman PDE

$$
\begin{gathered}
v_{t}+r(y) x v_{x}+a(z) v_{y}+\frac{1}{2} v_{y y} \\
+\operatorname{Sup}_{\pi_{t}, c}\left\{\frac{1}{2} \pi_{t}^{2} \sigma^{2}(z) v_{x x}+\left[\pi_{t}(\mu(z)-r(z))-c_{t}\right] v_{x}\right. \\
\left.+\gamma \rho \sigma(z) \pi_{t} v_{x y}+u_{2}\left(c_{t}\right)\right\}=0, \\
v(T, x, z)=U(x),
\end{gathered}
$$

Hence, the optimal solutions are

$$
\pi_{t}^{*}=-\frac{(\mu(z)-r(z)) v_{x}}{\sigma^{2}(z) v_{x x}}-\frac{\gamma \rho v_{x y}}{\sigma(z) v_{x x}},
$$




$$
U_{2}^{\prime}\left(c_{t}^{*}\right)=v_{x}
$$

Using the result of Alghalith [3], the optimal portfolio can be expressed as

$$
\pi_{t}^{*}=-\frac{(\mu(z)-r(z))\left(\alpha_{1}+\alpha_{2} c_{t}^{*}\right)}{\sigma^{2}(z)}-\frac{\alpha_{3} \rho}{\sigma(z)},
$$

and thus

$$
\mu(z)=r-\frac{\pi_{t}^{*} \sigma^{2}(z)+\alpha_{3} \rho \sigma(z)}{\left(\alpha_{1}+\alpha_{2} c_{t}^{*}\right)}
$$

where $\alpha_{i}$ is a constant that can be estimated. Moreover, this formula allows us to determine the impact of consumption on the rate of the return of the portfolio, as follows

$$
\frac{\partial \mu(z)}{\partial c_{t}^{*}}=\frac{\alpha_{2}(\mu(z)-r(z))\left(\alpha_{1}+\alpha_{2} c_{t}^{*}\right)+\alpha_{2}\left(\pi_{t}^{*} \sigma^{2}(z)+\alpha_{3} \rho \sigma(z)\right)}{\left(\alpha_{1}+\alpha_{2} c_{t}^{*}\right)^{2}}
$$

\section{Empirical Example}

We used quarterly data for Jamaica for the period March 1998 to June 2010 for real private aggregate consumption (in millions of dollars), stock index (JSI) and the Treasury bill rate $(r)$, and GDP (as the stochastic factor $Z_{s}$ ). We also computed the volatility of the index and the correlation factor between GDP and the JSI.

Using ()$-()$, we estimated each of the following non-linear equations

$$
\begin{gathered}
\pi_{t}^{*}=-\frac{(\mu(z)-r(z))\left(\beta_{1}+\beta_{2} c_{t}^{*}\right)}{\sigma^{2}(z)}-\frac{\beta_{3} \rho}{\sigma(z)}+\varepsilon_{1}, \\
\mu(z)=r-\frac{\pi_{t}^{*} \sigma^{2}(z)+\beta_{4} \rho \sigma(z)}{\beta_{5}+\beta_{6} c_{t}^{*}}+\varepsilon_{3},
\end{gathered}
$$

where $\beta_{i} s$ are the parameter to be estimated, while the other variables are observed data, and $\varepsilon$ is the esti- mation error. Using the estimated values $\hat{\beta}_{i} s$, we obtain the following comparative statics

$$
\frac{\partial \mu(z)}{\partial c_{t}^{*}}=\frac{\hat{\beta}_{2}(\mu(z)-r(z))\left(\hat{\beta}_{5}+\hat{\beta}_{6} c_{t}^{*}\right)+\hat{\beta}_{6}\left(\pi_{t}^{*} \sigma^{2}(z)+\hat{\beta}_{4} \rho \sigma(z)\right)}{\left(\hat{\beta}_{5}+\hat{\beta}_{6} c_{t}^{*}\right)^{2}} .
$$

In contrast, to previous studies that used simple linear regressions, the results support the existence of a very weak relationship between private consumption in Jamaica and the rate of return of the stock index (see

Table 1. Empirical results.

\begin{tabular}{cccc}
\hline$\hat{\beta}_{2}$ & 502072.4 & $\hat{\beta}_{6}$ & -36904.06 \\
$\hat{\beta}_{4}$ & $-1.67 \mathrm{E}+12$ & & \\
$\hat{\beta}_{5}$ & 29470778 & $\frac{\partial \mu(z)}{\partial c_{t}^{*}}$ & $3.78 \mathrm{E}-11$ \\
\hline
\end{tabular}

Table 1).

\section{References}

[1] J. Cvitanic and F. Zapatero, "Introduction to the Economics and Mathematics of Financial Markets," MIT Press, Cambridge, 2004.

[2] M. Alghalith, "A New Stochastic Factor Model: General Explicit Solutions," Applied Mathematics Letters, Vol. 22, No. 12, 2009, pp. 1852-1854. doi:10.1016/j.aml.2009.07.011

[3] M. Alghalith, "General Closed-Form Solutions to the Dynamic Optimization Problem in Incomplete Markets," 2011. http://mpra.ub.uni-muenchen.de/21950/ 